\title{
LARGE DEVIATIONS ANALYSIS FOR THE DETECTION OF 2D HIDDEN GAUSS-MARKOV RANDOM FIELDS USING SENSOR NETWORKS
}

\author{
Youngchul Sung ${ }^{\dagger}$, H. Vincent Poor and Heejung Yu
}

\begin{abstract}
The detection of hidden two-dimensional Gauss-Markov random fields using sensor networks is considered. Under a conditional autoregressive model, the error exponent for the Neyman-Pearson detector satisfying a fixed level constraint is obtained using the large deviations principle. For a symmetric first order autoregressive model, the error exponent is given explicitly in terms of the SNR and an edge dependence factor (field correlation). The behavior of the error exponent as a function of correlation strength is seen to divide into two regions depending on the value of the SNR. At high SNR, uncorrelated observations maximize the error exponent for a given SNR, whereas there is non-zero optimal correlation at low SNR. Based on the error exponent, the energy efficiency (defined as the ratio of the total information gathered to the total energy required) of ad hoc sensor network for detection is examined for two sensor deployment models: an infinite area model and and infinite density model. For a fixed sensor density, the energy efficiency diminishes to zero at rate $O\left(\right.$ area $\left.^{-1 / 2}\right)$ as the area is increased. On the other hand, non-zero efficiency is possible for increasing density depending on the behavior of the physical correlation as a function of the link length.
\end{abstract}

Index Terms- Neyman-Pearson detection, error exponent, GMRF

\section{INTRODUCTION}

Consider the design of a sensor network for the detection of a correlated stochastic signal in a fixed area. Many questions arise in such a design: How do the field correlation and measurement signal-to-noise (SNR) affect the detection performance? What is the optimal sensor density, i.e., the number of nodes per unit area? What is the information and energy trade-off in such a sensor network with ad hoc routing? To address these issues, several studies based on one-dimensional (1D) spatial signal models have been conducted (see, e.g., [1] and [2]). However, there is an important difference between 1D signal models and actual spatial signals. Suppose that we take observations from sensors placed equidistantly along a line transect laid over a given area. The observations may then be viewed as samples generated by a one-dimensional process and the results from time series analysis could be applied to investigate their statistical properties. However, there is no real notion of 'signal flow' or dependence direction along the transect as there is in a more traditionally obtained time series. For samples from sensors deployed over a two-dimensional (2D) area, it is necessary to consider the signal dependence in all direction in the plane, and as a consequence, answering the above questions becomes more difficult.

To address the above questions in a 2D setting, in this paper, we consider the detection of 2D Gauss-Markov random fields

\footnotetext{
${ }^{\dagger}$ Y. Sung and H. Yu are with the Dept. of Electrical Engineering, Korea Advanced Institute of Science and Technology (KAIST), Daejeon 305-701, South Korea. Email:ysung@ee.kaist.ac.kr and hjyu@stein.kaist.ac.kr. H. V. Poor is with the Dept. of Electrical Engineering, Princeton University, Princeton, NJ 08544. Email: poor@princeton.edu. The work of Y. Sung was supported in part by Brain Korea 21 Project, the School of Information Technology, KAIST. The work of H. V. Poor was supported in part by the U. S. National Science Foundation under Grants ANI-0338807 and CNS-06-25637.
}

(GMRFs) using noisy observations. In particular we consider Sensors $i j$ located on a 2D lattice $\mathcal{I}$. On denoting the (noisy) measurements of Sensor $i j$ as $Y_{i j}$ and adopting a Neyman-Pearson formulation, we can model the detection problem via null and alternative hypotheses given by

$$
\mathcal{H}_{0}: Y_{i j}=W_{i j}, i j \in \mathcal{I} \text { vs. } \quad \mathcal{H}_{1}: Y_{i j}=X_{i j}+W_{i j}, i j \in \mathcal{I},(1)
$$

where $\left\{W_{i j}\right\}$ represents independent and identically distributed (i.i.d.) $\mathcal{N}\left(0, \sigma^{2}\right)$ noise with a known variance $\sigma^{2}$, and $\left\{X_{i j}\right\}$ is a stationary GMRF on the 2D lattice $\mathcal{I}$ independent of the measurement noise $\left\{W_{i j}\right\}$. Thus, the observation samples form a $2 \mathrm{D}$ hidden GMRF under $\mathcal{H}_{1}$.

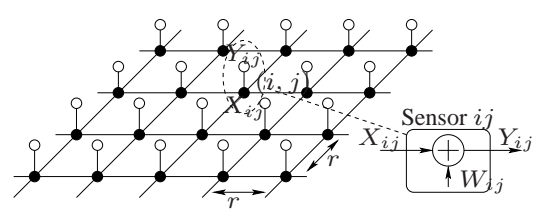

Fig. 1. Sensors on a 2D Lattice $\mathcal{I}$ : Hidden Markov Structure

\subsection{Summary of Results}

The exact error probability of the detection of the Neyman-Pearson test is not available in closed-form in the general correlated case, including the hypotheses 11 . Hence, we invoke the the large deviations principle and use the error exponent of the detection probability (or, more conveniently, its complement, the miss probability) as an alternative performance measure. For a fixed false-alarm level, the miss probability $P_{M}$ decays exponentially as the sample size $n$ increases, and the error exponent is defined as the rate of exponential decay, i.e.,

$$
\mathcal{K} \triangleq \lim _{n \rightarrow \infty}-\frac{1}{n} \log P_{M}
$$

under the given constraint (i.e., the false alarm probability $P_{F} \leq$ $\alpha$ ). The error exponent is a good performance criterion in the large sample regime since it allows the designer to estimate the number of samples required for a given detection performance. Hence, efficient design can be examined through the error exponent for large scale sensor networks.

Here, we adopt the conditional autoregression (CAR) model for the signal, and derive a closed-form expression for the error exponent $\mathcal{K}$ of the miss probability (which is independent of $\alpha$ ) in the spectral domain. We do so by exploiting the spectral structure of the CAR signal and the relationship between the eigenvalues of the block circulant approximation to a block Toeplitz matrix describing the 2D correlation structure. In particular, it is shown that the error exponent for the detection of 2D hidden GMRF is an extension of that in the 1D case obtained by Sung et al.[3]. As in the $1 \mathrm{D}$ case, it is shown that i.i.d. (and, thus, uncorrelated) observations maximize the error exponent for a given SNR when the SNR is high. On the other hand, there is an optimal non-zero degree of 
correlation at low SNR. Interestingly, it is seen that there is a discontinuity in the optimal correlation strength as a function of SNR. In the perfectly correlated case, the error exponent is zero as expected. For the error exponent as a function of SNR, we will show that the error exponent increases as $\log$ SNR for a given correlation strength at high SNR.

We consider two asymptotic regimes modelling the sensor deployment in 2D: an infinite area model with a fixed density and an infinite density model with a fixed area. Applying the results, we obtain the asymptotic behavior of the energy efficiency, defined as the ratio of the total information gathered to the required energy to obtain information from the area for an ad hoc network with minimum hop routing to the fusion center. For the infinite area model, the energy efficiency decays to zero with rate $O\left(\right.$ area $\left.^{-1 / 2}\right)$ as we increase the coverage area. For the infinite density model, on the other hand, a non-zero efficiency is possible if the decay rate of the error exponent $\mathcal{K}$ (density) as a function of density is slower than $O\left(\right.$ density $\left.^{(1-\delta) / 2}\right)$, where $\delta$ is the propagation constant $\delta \geq 2$.

\subsection{Related Work}

The detection of Gauss-Markov processes in Gaussian noise is a classical problem. See [4] and references therein. However, most work in this area considers only 1D signals or time series. A closed-form error exponent was obtained and its properties were investigated for 1D hidden Gauss-Markov random processes [3]. Large deviations analyses were used to examine the issues of optimal sensor density and optimal sampling were examined with a 1D signal model in [1] and [2].

An error exponent was obtained for the detection of 2D GMRFs in [5], where the sensors are located randomly and the Markov graph is based on the nearest neighbor dependency enabling a loop-free graph and further analysis. In this work, however, the measurement noise was not captured. Our work here focuses on the error exponent for the detection of 2D hidden GMRF on a 2D infinite lattice, which allows for the consideration of measurement noise. In particular we examine the above CAR model and investigate of the detection performance with respect (w.r.t.) to various design parameters such as correlation strength, measurement SNR, sensor density and area.

\section{DATA MODEL}

Definition 1 (GMRF [6]) A random vector $\mathbf{X}=\left(X_{1}, X_{2}, \cdots, X_{n}\right)$ $\in \mathbb{R}^{n}$ is a Gauss-Markov random field w.r.t. a labelled graph $\mathcal{G}=(\nu, \mathcal{E})$ with mean $\boldsymbol{\mu}$ and precision matrix $\mathbf{Q}>0$, if its probability density function is given by

$$
p(\mathbf{X})=(2 \pi)^{-n / 2}|\mathbf{Q}|^{1 / 2} \exp \left(-\frac{1}{2}(\mathbf{X}-\boldsymbol{\mu})^{T} \mathbf{Q}(\mathbf{X}-\boldsymbol{\mu})\right),
$$

and $Q_{l m} \neq 0 \Longleftrightarrow\{l, m\} \in \mathcal{E}$ for all $l \neq m$. Here, $\nu$ is the set of all nodes $\{1,2, \cdots, n\}$ and $\mathcal{E}$ is the set of edges connecting pairs of nodes, which represent the conditional dependence structure.

Note that the mean and the precision matrix fully characterize a GMRF. Note also that the covariance matrix $\mathbf{Q}^{-1}$ is completely dense in general while the precision matrix $\mathbf{Q}$ has nonzero elements $Q_{l m}$ only when there is an edge between nodes $l$ and $m$ in the Markov random field. Hence, when the graph is not fully connected, the precision matrix is sparse. The 2D indexing scheme $(i, j)$ can be properly converted to an 1D scheme to apply Definition 1 From here on, we use the 2D indexing scheme for convenience.

Definition 2 (Stationarity) A $2 D$ GMRF on $2 D$ doubly infinite lattice $\mathcal{I}_{\infty}$ is said to be stationary if the mean vector is constant and $\operatorname{Cov}\left(X_{i j}, X_{i^{\prime} j^{\prime}}\right) \triangleq \mathbb{E}\left\{X_{i j} X_{i^{\prime} j^{\prime}}\right\}=c\left(i-i^{\prime}, j-j^{\prime}\right)$ for some function $c(\cdot, \cdot)$.

For a 2D stationary GMRF $\left\{X_{i j}\right\}$, the covariance $\left\{\gamma_{i j}\right\}$ is defined as

$$
\gamma_{i j}=\mathbb{E}\left\{X_{i^{\prime} j^{\prime}} X_{i^{\prime}+i, j^{\prime}+j}\right\}=\mathbb{E}\left\{X_{00} X_{i j}\right\},
$$

which does not depend on $i^{\prime}$ or $j^{\prime}$ due to the stationarity. Further, the spectral density function of a zero-mean and stationary Gaussian process $\left\{X_{i j}\right\}$ on $\mathcal{I}_{\infty}$ with covariance $\gamma_{i j}$ is defined as

$$
f\left(\omega_{1}, \omega_{2}\right)=\frac{1}{4 \pi^{2}} \sum_{i j \in \mathcal{I}_{\infty}} \gamma_{i j} \exp \left(-\iota\left(i \omega_{1}+j \omega_{2}\right)\right),
$$

where $\iota=\sqrt{-1}$ and $\left(\omega_{1}, \omega_{2}\right) \in(-\pi, \pi]^{2}$. Note that this is a $2 \mathrm{D}$ extension of the conventional 1D discrete-time Fourier transform (DTFT).

Definition 3 (The conditional autoregression (CAR)) A GMRF can be specified using a set of full conditional normal distributions with mean and precision:

$$
\begin{aligned}
\mathbb{E}\left\{X_{i j} \mid \mathbf{X}_{-i j}\right\} & =-\frac{1}{\theta_{00}} \sum_{i^{\prime} j^{\prime} \in \mathcal{I}_{\infty} \neq 00} \theta_{i^{\prime} j^{\prime}} X_{i+i^{\prime}, j+j^{\prime}}, \\
\operatorname{Prec}\left\{X_{i j} \mid \mathbf{X}_{-i j}\right\} & =\theta_{00}>0,
\end{aligned}
$$

where $\mathbf{X}_{-i j}$ denotes the set of all variables except $X_{i j}$.

It is shown that the GMRF defined by the CAR model (6) - (7) is a zero-mean stationary Gaussian process on $\mathcal{I}_{\infty}$ with the spectral density function [6]

$$
f\left(\omega_{1}, \omega_{2}\right)=\frac{1}{4 \pi^{2}} \frac{1}{\sum_{i j \in \mathcal{I}_{\infty}} \theta_{i j} \exp \left(-\iota\left(i \omega_{1}+j \omega_{2}\right)\right)}
$$

if

$$
\begin{aligned}
& \left|\left\{\theta_{i j} \neq 0\right\}\right|<\infty, \quad \theta_{i j}=\theta_{-i,-j}, \quad \theta_{00}>0 \\
& \left\{\theta_{i j}\right\} \text { is so that } f\left(\omega_{1}, \omega_{2}\right)>0, \quad \forall\left(\omega_{1}, \omega_{2}\right) \in(-\pi, \pi]^{2} .(10)
\end{aligned}
$$

We assume that the 2D stochastic signal in 11 is given by a stationary GMRF defined by the CAR model (6) - 7) and (9) - 10. Then, the observation spectrum under the two hypotheses (1) are given, respectively, by

$$
S_{0}^{y}\left(\omega_{1}, \omega_{2}\right)=\frac{\sigma^{2}}{4 \pi^{2}} \text { and } S_{1}^{y}\left(\omega_{1}, \omega_{2}\right)=\frac{\sigma^{2}}{4 \pi^{2}}+f\left(\omega_{1}, \omega_{2}\right) .
$$

\section{PERFORMANCE MEASURE: ERROR EXPONENT}

In this section, we investigate the performance of the NeymanPearson detector with level $\alpha \in(0,1)$ for a 2D CAR signal in noisy observations. We obtain the error exponent in the spectral domain for this problem by exploiting the spectral structure of the CAR signal and the relationship between the eigenvalues of block circulant and block Toeplitz matrices representing 2D correlation structure.

Theorem 1 (Error Exponent) Consider Neyman-Pearson detection between the hypotheses (1) with the model (6) - (7) and with level $\alpha \in(0,1)$. Assuming that conditions $(9$ and 10 hold, the error exponent of the miss probability is independent of $\alpha$ and is given by

$$
\begin{aligned}
\mathcal{K}= & \frac{1}{4 \pi^{2}} \int_{-\pi}^{\pi} \int_{-\pi}^{\pi}\left(\frac{1}{2} \log \frac{\sigma^{2}+4 \pi^{2} f\left(\omega_{1}, \omega_{2}\right)}{\sigma^{2}}\right. \\
& \left.\quad+\frac{1}{2} \frac{\sigma^{2}}{\sigma^{2}+4 \pi^{2} f\left(\omega_{1}, \omega_{2}\right)}-\frac{1}{2}\right) d \omega_{1} d \omega_{2}, \quad(11) \\
= & \frac{1}{4 \pi^{2}} \int_{-\pi}^{\pi} \int_{-\pi}^{\pi} D\left(\mathcal{N}\left(0, S_{0}^{y}\left(\omega_{1}, \omega_{2}\right)\right) \| \mathcal{N}\left(0, S_{1}^{y}\left(\omega_{1}, \omega_{2}\right)\right) d \omega_{1} d \omega_{2},\right.
\end{aligned}
$$

where $D(\cdot \| \cdot)$ denotes the Kullback-Leibler divergence. 
Proof: $\mathcal{K}$ is given by the almost-sure limit of the asymptotic Kullback-Leibler rate $\mathcal{K}=\lim _{n \rightarrow \infty} \frac{1}{n} \log \frac{p_{0, n}}{p_{1, n}}\left(\mathbf{y}_{n}\right)$ evaluated under $p_{0, n}[7]$. Using the fact that we have Gaussian distributions under both hypotheses, we have

$$
\mathcal{K}=\lim _{n \rightarrow \infty} \frac{1}{n}\left(\frac{1}{2} \log \frac{\operatorname{det}\left(\boldsymbol{\Sigma}_{1, n}\right)}{\operatorname{det}\left(\boldsymbol{\Sigma}_{0, n}\right)}+\frac{1}{2} \mathbf{y}_{n}^{T}\left(\boldsymbol{\Sigma}_{1, n}^{-1}-\boldsymbol{\Sigma}_{0, n}^{-1}\right) \mathbf{y}_{n}\right),
$$

Then approximating the block Toeplitz correlation matrix with a block circulant matrix and applying the the 2D Grenander-Szegö theorem, we obtain the limit of each term as follows.

$$
\begin{aligned}
\frac{1}{n} \log \operatorname{det}\left(\boldsymbol{\Sigma}_{1, n}\right) & \rightarrow \frac{1}{4 \pi^{2}} \int_{-\pi}^{\pi} \int_{-\pi}^{\pi} \log \left(\sigma^{2}+4 \pi^{2} f\left(\omega_{1}, \omega_{2}\right)\right) d \omega_{1} d \omega_{2}, \\
\frac{1}{n} \log \operatorname{det}\left(\boldsymbol{\Sigma}_{0, n}\right) & \rightarrow \log \sigma^{2}, \\
\frac{1}{n} \mathbf{y}_{n}^{T} \boldsymbol{\Sigma}_{1, n}^{-1} \mathbf{y}_{n} & \rightarrow \frac{1}{4 \pi^{2}} \int_{-\pi}^{\pi} \int_{-\pi}^{\pi} \frac{\sigma^{2}}{\sigma^{2}+4 \pi^{2} f\left(\omega_{1}, \omega_{2}\right)} d \omega_{1} d \omega_{2}, \\
\frac{1}{n} \mathbf{y}_{n}^{T} \boldsymbol{\Sigma}_{0, n}^{-1} \mathbf{y}_{n} & \rightarrow 1,
\end{aligned}
$$

almost surely.

This theorem is a $2 \mathrm{D}$ extension of the error exponent of $1 \mathrm{D}$ hidden Gauss-Markov model based on state-space structure obtained in [3]. Intuitively, the error exponent (11] can be explained using the frequency binning argument. For each $2 \mathrm{D}$ frequency segment $d \omega_{1} d \omega_{2}$, the spectra are flat, i.e., the signals are independent and Stein's lemma can be applied for the segment. The overall Kullback-Leibler divergence is the sum of contributions from each bin.

\subsection{Symmetric First Order Autoregression}

To investigate the behavior of the error exponent as a function of correlation and SNR, we further consider the symmetric first order autoregression (SFAR), described by the conditions

$$
\begin{aligned}
\mathbb{E}\left\{X_{i j} \mid \mathbf{X}_{-i j}\right\} & =\frac{\lambda}{\kappa}\left(X_{i+1, j}+X_{i-1, j}+X_{i, j+1}+X_{i, j-1}\right), \\
\operatorname{Prec}\left\{X_{i j} \mid \mathbf{X}_{-i j}\right\} & =\kappa>0,
\end{aligned}
$$

where $0 \leq \lambda \leq \frac{\kappa}{4}$. (This is a sufficient condition to satisfy 9) 101.) Note here that $\theta_{00}=\kappa$ and $\theta_{1,0}=\theta_{-1,0}=\theta_{0,1}=\theta_{0,-1}=$ $-\lambda$. In this model, the correlation is symmetric for each set of four neighboring nodes. The SFAR model is a simple yet meaningful extension of the 1D Gauss-Markov random process, which has the conditional causal dependency only on the previous sample. Here in the 2D case we have four neighboring nodes in the four (planar) directions. The spectrum of the SFAR is given by

$$
f\left(\omega_{1}, \omega_{2}\right)=\frac{1}{4 \pi^{2} \kappa\left(1-2 \zeta \cos \omega_{1}-2 \zeta \cos \omega_{2}\right)} .
$$

We define the edge dependence factor $\zeta$ by

$$
\zeta \triangleq \frac{\lambda}{\kappa}, \quad 0 \leq \zeta \leq 1 / 4
$$

Note that $\zeta=0$ corresponds to the i.i.d. case whereas $\zeta=1 / 4$ corresponds to the perfectly correlated case. Hence, the correlation strength can be captured in this single quantity $\zeta$ for SFAR signals. The power of the SFAR is obtained using the inverse Fourier transform via the relation (5), and is given by

$$
P_{s}=\gamma_{00}=\frac{2 K(4 \zeta)}{\pi \kappa}, \quad\left(0 \leq \zeta \leq \frac{1}{4}\right),
$$

where $K(\cdot)$ is the complete elliptic integral of the first kind [8]. The SNR is given by SNR $=\frac{P_{s}}{\sigma^{2}}=\frac{2 K(4 \zeta)}{\pi \kappa \sigma^{2}}$. Using eq. (11) and the SNR, we obtain the error exponent in the SFAR signal case, denoted by $\mathcal{K}_{s}$ and given in the following corollary.
Corollary 1 The error exponent for the Neyman-Pearson detector for the hypotheses (17) with the SFAR 2D signal model is given by

$$
\begin{aligned}
\mathcal{K}_{S}=\frac{1}{4 \pi^{2}} \int_{-\pi}^{\pi} \int_{-\pi}^{\pi}\left(\frac{1}{2} \log \left(1+\frac{S N R}{(2 / \pi) K(4 \zeta)\left(1-2 \zeta \cos \omega_{1}-2 \zeta \cos \omega_{2}\right)}\right)\right. \\
\left.+\frac{1}{2} \frac{1}{1+\frac{1}{(2 / \pi) K(4 \zeta)\left(1-2 \zeta \cos \omega_{1}-2 \zeta \cos \omega_{2}\right)}}-\frac{1}{2}\right) d \omega_{1} d \omega_{2} \cdot
\end{aligned}
$$

Note that the SNR and correlation are separated in (15), which enables us to investigate the effects of each term separately.

\subsection{Properties of the Error Exponent $\mathcal{K}_{s}$}

First, it is readily seen from Corollary 1 that $\mathcal{K}_{s}$ is a continuous function of the edge dependence factor $\zeta(0 \leq \zeta \leq 1 / 4)$ for a given SNR. The values of $\mathcal{K}_{s}$ at the extreme correlations are given by noting that $K(0)=\frac{\pi}{2}$ and $K(1)=\infty$. Therefore, in the i.i.d. case (i.e., $\zeta=0$ ), the corollary reduces to Stein's lemma as expected, and $\mathcal{K}_{s}$ is given by

$\mathcal{K}_{s}=\frac{1}{2} \log (1+\mathrm{SNR})+\frac{1}{2(1+\mathrm{SNR})}-\frac{1}{2}=D\left(\mathcal{N}\left(0, \sigma^{2}\right) \| \mathcal{N}\left(0, \sigma^{2}+P_{s}\right)\right)$.

For the perfectly correlated case $(\zeta=1 / 4)$, on the other hand, $\mathcal{K}_{s}=0$. In fact, in this case as well as in the i.i.d. case, the two-dimensionality is irrelevant. The known result that $P_{M} \sim$ $\Theta\left(n^{-1 / 2}\right)$ for the perfectly correlated case is applicable.

For intermediate values of correlation, we evaluate (15) for several different SNR values, as shown in Fig. 2 It is seen that at

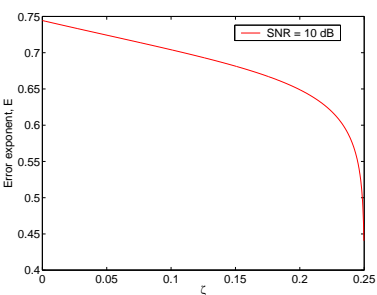

(a)

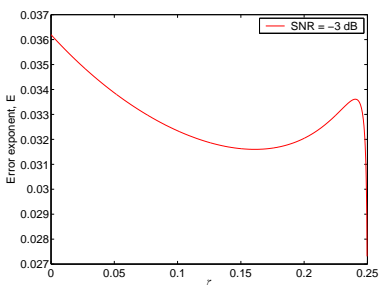

(c)

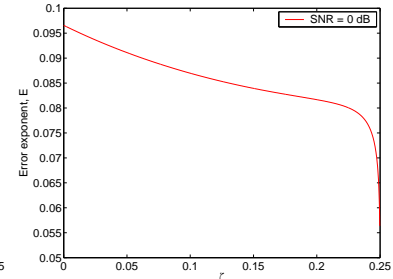

(b)

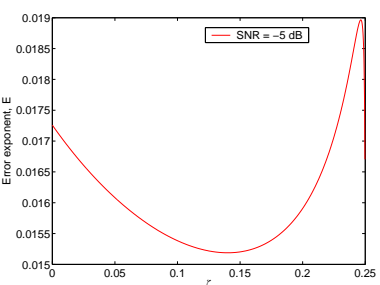

(d)
Fig. 2. $\mathcal{K}_{s}$ as a function of $\zeta$ : (a) $\mathrm{SNR}=10 \mathrm{~dB}$, (b) $\mathrm{SNR}=0 \mathrm{~dB}$, (c) $\mathrm{SNR}=-3 \mathrm{~dB}$, (d) $\mathrm{SNR}=-5 \mathrm{~dB}$

high SNR $\mathcal{K}_{s}$ is monotonically decreasing as $\zeta$ increases. Hence, i.i.d. observations give the best error performance for a given value of SNR when SNR is large, as in the 1D case [3]. As we decrease the SNR, it is observed that a second mode grows near $\zeta=1 / 4$. As we further decrease the SNR, the value of $\zeta$ of the second mode shifts toward $1 / 4$, and the value of the second mode exceeds that of the i.i.d. case. Hence, there is a discontinuity in the optimal correlation as a function of SNR in the $2 \mathrm{D}$ case even if the maximal $\mathcal{K}_{s}$ itself is continuous. This is not the case in 1D.

With regard to $\mathcal{K}_{s}$ as a function of SNR, it is straightforward to see that it is continuous and increases at the rate log SNR at high SNR for a given value of $\zeta$. 


\section{AD HOC NETWORKING: INFORMATION-ENERGY TRADE-OFF}

The analytical results in the previous section can be applied to answer some fundamental questions in the design of sensor networks for detection applications. We consider a planar ad hoc sensor network with minimum hop routing. To simplify the analysis, we assume that $(2 n+1)^{2}$ sensors are located on the grid $[-n: 1: n]^{2}$ with spacing $r_{n}$, as shown in Fig. 1 and a fusion center is located at the center $(0,0)$. We assume that the measurement $Y_{i j}$ is delivered to the fusion center using the minimum hop routing, which requires a hop count of $|i|+|j|$.

\subsection{Physical correlation model}

The actual physical correlation in this model can be obtained by solving a proper continuous index $2 \mathrm{D}$ stochastic differential equation (SDE), e.g.,

$$
\left[\left(\frac{\partial}{\partial x}\right)^{2}+\left(\frac{\partial}{\partial y}\right)^{2}-\xi^{2}\right] X(x, y)=u(x, y)
$$

where $u(x, y)$ is the process noise and $\xi$ is a parameter determining the correlation strength of the field. By solving a proper SDE, the edge correlation factor $\rho$ is given, as a function of the edge length $r_{n}$, by

$$
\rho=f\left(r_{n}\right) .
$$

Typically, $f(\cdot)$ is a positive and monotonically decreasing function of $r_{n}$. Further, we have a monotone mapping $g: \rho \rightarrow \zeta$ from the edge correlation factor $\rho$ to the edge dependence factor $\zeta$, which maps zero and one to zero and $1 / 4$, respectively. Thus, we have $\zeta=g\left(f\left(r_{n}\right)\right.$ ), and for given physical parameters (with a slight abuse of notation),

$$
\mathcal{K}_{s}(\mathrm{SNR}, \zeta)=\mathcal{K}_{s}\left(\mathrm{SNR}, g\left(f\left(r_{n}\right)\right)\right)=\mathcal{K}_{s}\left(\mathrm{SNR}, r_{n}\right) .
$$

We will use the arguments SNR and $\zeta$ for $\mathcal{K}_{s}$ properly if necessary.

\subsection{Energy efficiency}

We now consider the energy efficiency of the ad hoc sensor network as the network size grows. The energy efficiency $\eta$ can be defined as

$$
\eta=\frac{\text { total gathered information } I_{t}}{\text { total required energy } E_{t}},
$$

where $I_{t}$ is given by the product of the number of sensors and the information $\mathcal{K}_{s}$ per each sensor. We consider two asymptotic regimes for the increase in network size: an infinite area model with fixed density and an infinite density model with fixed area. The behavior of the energy efficiency as we increase the network size is summarized in the following theorems.

Theorem 2 (Infinite area model) For an ad hoc sensor network with increasing area and a fixed node density, the energy efficiency decays to zero as we increase the area with rate

$$
\eta=O\left(\text { area }^{-1 / 2}\right) \text {. }
$$

Proof: The total energy required for data gathering is given by $E_{t}=E_{l i n k}\left(r_{n}\right) \sum_{i=-n}^{n} \sum_{j=-n}^{n}(|i|+|j|)=2 n(n+1)(2 n+1) E_{l i n k}\left(r_{n}\right)$, where the transmission energy per link $E_{\text {link }}\left(r_{n}\right)=r_{n}^{\delta}$ and $\delta$ is the propagation loss factor. We have $I_{t}=(2 n+1)^{2} \mathcal{K}_{s}\left(r_{n}\right)$, and area $=\Theta\left(n^{2}\right)$. The energy efficiency is given by

$$
\eta=\frac{(2 n+1)^{2} \mathcal{K}_{s}\left(r_{n}\right)}{2 n(n+1)(2 n+1) E_{\text {link }}\left(r_{n}\right)} .
$$

Since $r_{n}$ is fixed, $\mathcal{K}_{s}$ and $E_{\text {link }}$ do not change with $n$, and 17. follows.

Theorem 3 (Infinite density model) For the infinite density model, a non-zero efficiency is possible if the decay rate of the error exponent $\mathcal{K}_{s}$ as a function of density is slower than

$$
O\left(\text { density }^{(1-\delta) / 2}\right) \text {. }
$$

Proof: For the infinite density model, we have

$$
r_{n}=\Theta\left(n^{-1}\right), r_{n}^{\delta}=\Theta\left(n^{-\delta}\right), \text { density }=\Theta\left(n^{2}\right) .
$$

From 18, we have $\eta=\mathcal{K}_{s}\left(r_{n}\right) / n^{1-\delta}$. If $\mathcal{K}_{s}$ as a function of $r_{n}$ decays slower than $n^{1-\delta}, \eta$ does not diminish to zero.

The non-zero efficiency in the asymptotic regime depends on the decay rate of $\mathcal{K}_{s}$ as a function of $r_{n}$. Since $\mathcal{K}_{s}(\zeta)$ is given, this depends on the functions $f$ and $g$ in Section 4.1 and the propagation loss factor $\delta$.

\section{CONCLUSIONS}

We have considered the detection of 2D GMRFs from noisy observations. We have adopted the CAR model for the signal, and have used a spectral domain approach to derive the error exponent for the Neyman-Pearson detector satisfying a fixed level constraint. Under the symmetric first order autoregressive model, we have obtained the error exponent explicitly in terms of the SNR and the edge dependence factor. We have investigated the properties of the error exponent as a function of SNR and correlation. We have seen that the behavior of the error exponent w.r.t. correlation strength is divided into two regions depending on SNR. At high SNR, i.i.d. (and, thus, uncorrelated) observations maximize the error exponent for a given SNR, whereas there is non-zero optimal value of correlation at low SNR. Further, it has been seen that there is a discontinuity for the optimal correlation as a function of SNR. Based on the error exponent, we have also investigated the energy efficiency of ad hoc sensor network for detection applications. For a fixed node density, the energy efficiency decays to zero with rate $O\left(\operatorname{area}^{-1 / 2}\right)$ as we increase the area. On the other hand, non-zero efficiency is possible with increasing density depending on physical correlation strength as a function of the link length.

\section{REFERENCES}

[1] Y. Sung, L. Tong and H. V. Poor, "Sensor configuration and activation for field detection in large sensor arrays," in Proc. 2005 Information Processing in Sensor Networks (IPSN), Los Angeles, CA, Apr. 2005.

[2] J.-F. Chamberland and V. V. Veeravalli, "How dense should a sensor network be for detection with correlated observations?," IEEE Trans. Inform. Theory, vol. 52, pp. 5099 - 5106, Nov. 2006.

[3] Y. Sung, L. Tong and H. V. Poor, "Neyman-Pearson detection of Gauss-Markov signals in noise: Closed-form error exponent and properties," IEEE Trans. In form. Theory, vol. 52, pp. 1354 - 1365, Apr. 2006.

[4] T. Kailath and H. V. Poor, "Detection of stochastic processes," IEEE Trans. Inform. Theory, vol. 44, pp. 2230 - 2259, Oct. 1998.

[5] A. Anandkumar, L. Tong and A. Swami, "Detection of Gauss-Markov random field on nearest-neighbor graph," in Proc. 2007 IEEE International Conference on Acoustics, Speech, and Signal Processing (ICASSP'07), Hawaii, USA, Apr. 2007.

[6] H. Rue and L. Held, Gaussian Markov Random Fields: Theory and Applicatons, Chapman \& Hall/CRC, New York, 2005.

[7] I. Vajda, Theory of Statistical Inference and Information, Kluwer Academic Publishers, Dordrecht, 1989.

[8] J. Besag, "On a system of two-dimensional recurrence equations," Journal of the Royal Statistical Society. Series B, vol. 43, no. 3, pp. 302 - 309, 1981. 